\title{
A Comprehensive Study on Safety of New Energy Vehicles
}

\author{
Zhenpo Wang ${ }^{1}$, Zekun Zhang ${ }^{1,2}, \mathrm{Ni}_{\text {Lin }}{ }^{1, *}$, Xiang Zhang ${ }^{1,2}$, Peng Liu ${ }^{1, *}$ and Ye Wu ${ }^{1}$ \\ ${ }^{1}$ Beijing Institute of Technology, Beijing 100081, China \\ ${ }^{2}$ Advanced Technology Research Institute (Jinan), Beijing Institute of Technology, Jinan 250300, China
}

\begin{abstract}
New energy vehicles (NEVs) have become a fundamental part of transportation system. Performance of an NEV is hugely determined by batteries, motors, and embedded electric control units. In this paper, a comprehensive study that covers all these key components is presented. Mechanisms and characterizations of failures are given in detail. On top of these, algorithms for fault diagnosis are established based on big data of real-world NEVs with joint considerations of design flaws, usage behaviors, and environmental conditions. In this way, multiple types of faults can be detected ahead of time to avoid accident. Proposed methods have been verified by real-world operational data, indicating effectiveness while providing insights for NEV design optimization.
\end{abstract}

Keywords: New energy vehicles, Fault diagnosis, Big data, Battery, Motor, Electric control system.

\section{INTRODUCTION}

In recent years, pollution and shortage in fuel supply have become increasingly serious, leading to a global consensus to develop new energy vehicles (NEV). In 2020, 3.125 million NEVs have been sold worldwide, increased by $41 \%$ over the previous year, and the total number of NEVs exceeded 10 million. Meanwhile, major automobile countries, including United States, Germany and Japan, have proposed national strategies of gradually replacing traditional fuel vehicles with NEVs in 15-20 year.

Unfortunately, due to design and manufacturing imperfections as well as complicated operational and environmental conditions, faults and malfunctions such as spontaneous fire and loss of power have been frequently reported, which has aroused concerns about safety and reliability of NEVs. Take electric cars as an example, according to an annual report from China, power batteries, automotive electronics, and motors account for more than $90 \%$ of the total faults. Therefore, fault diagnosis of the power battery, drive motor and electronic control system are critical to ensure safety and reliability [1].

Detailed analysis shows internal short circuit, overheating, overcharging, and poor consistency are the major causes for battery thermal runaway [2]. For motors, inter-turn short circuit is the major electrical fault mode, which leads to considerable amount of heat generation in motor winding that could eventually burn

*Address correspondence to this author at National Engineering Laboratory for Electric Vehicles, Beijing Institute of Technology, Beijing 100081, China;

E-mail:6120200190@bit.edu.cn; bitliupeng@bit.edu.cn

This work was supported by the National Natural Science Foundation of China under Grant 2020YFB1600602. a motor, posing great threats to driving safety. Electronic control systems are considered as the brains of NEVs, where any software or hardware failure may lead to serious accidents such as loss of power or control. This paper presents a comprehensive study for fault diagnosis and health evaluation for key components in NEVs mostly using artificial intelligence and statistical analysis methods. Establishment and validations of algorithms are based on real-world vehicle operation data. Results show effectiveness of the proposed methods play a positive role in improving the safety of NEVs, which also contributes in improvements in vehicle design.

The rest of the paper is organized as follows. Section II provides basic information of the national big data platform of NEVs in China that serves as the data sources in this work. Section III presents detailed analysis of fault diagnosis method of lithium-ion batteries. Research on motor temperature prediction and over-temperature diagnosis is introduced in Section IV. Section V focuses on health assessment method for electronic control system on top of statistical analysis. In the end, Section VI concludes the paper.

\section{DATA SOURCE}

Data used in this paper is from the National Big Data Platform of Electric Vehicles in China, which collects data from NEVs in a real-time fashion. By November 2021, over 6 million NEVs from 331 manufactures have been connected in. Roughly two types of data are collected. The first type is related to basic information of vehicles, such as vehicle type, manufacturer, mileage, speed, and so forth. Whereas 


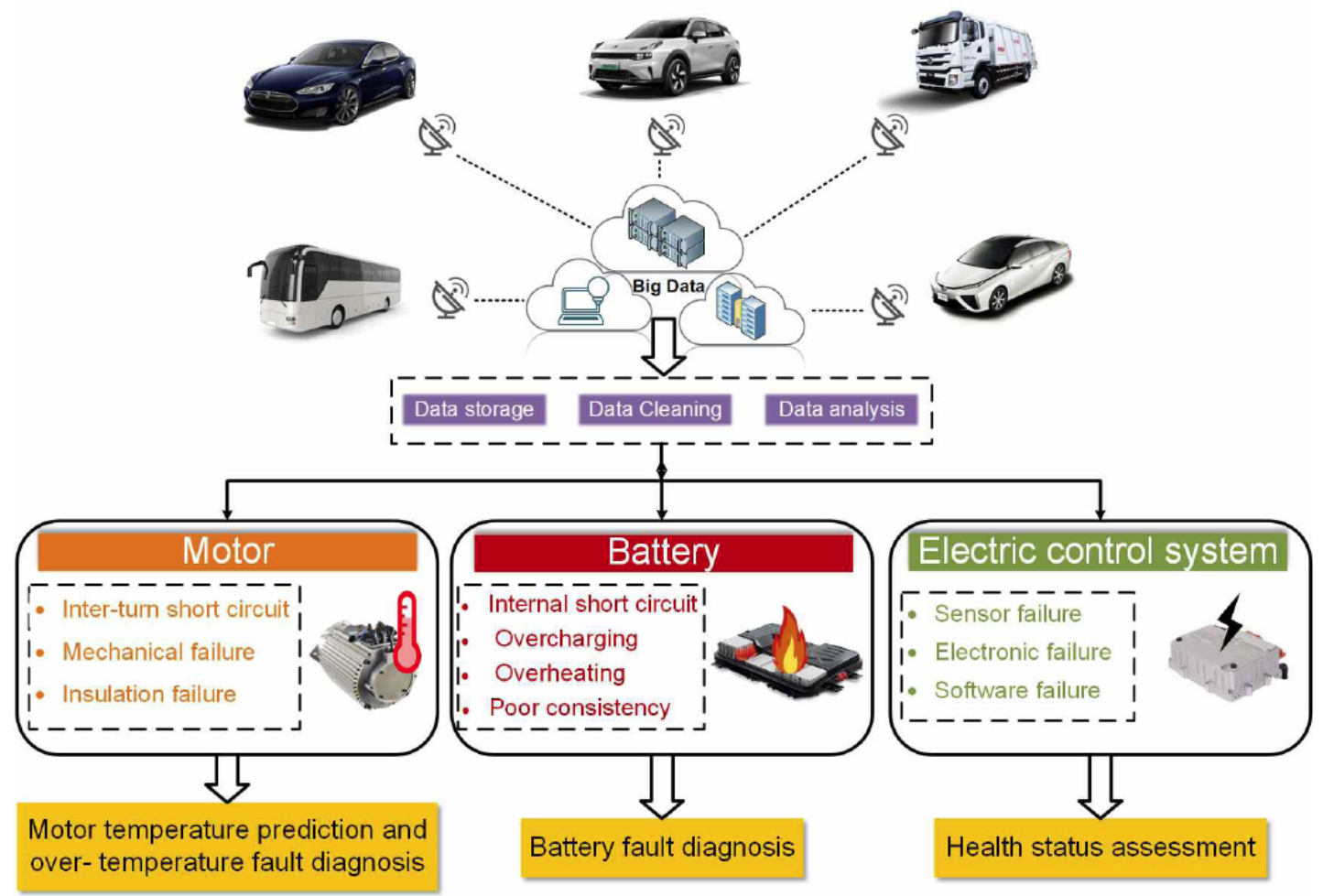

Figure 1: Overall structure of the system.

the second type involves operational data, including current, voltage, state of charge (SOC) of batteries, motor speed, torque, temperature, DC-DC alarm status, and so forth. In this work, data from more than 1000 vehicles were extracted and preprocessed for algorithm design and validation purposes.

Due to complexity in vehicle operational conditions, differences in usage behaviors, and instability in signal transmission, the data uploaded to the platform may contains noise or suffer from package lose. To solve these problems, data pre-processing is performed to ensure quality of data before algorithm establishment and verification.

\section{BATTERY}

In this work, we propose a novel method for battery fault diagnosis that covers sub-model selection, factor extraction and model fusion.

\subsection{Sub-models for Battery Fault Diagnosis}

In this work, sub-models refer to the models that could detect certain type of battery fault under a given condition. Three sub-models adopted for model fusion are introduced below.

\section{(1) Entropy-based model}

As indicated by its name, this model is established on top of entropy of battery voltage curves from the perspective of information theory. By combining Shannon entropy and Z-score, hidden information can be extracted so that numerical outlier and inconsistency among cells can be identified [3]. Together with safety threshold obtained through statistical analysis using big data, early warning of faults can be realized [4].

\section{(2) Voltage fluctuation based model}

This method detects faults through identification of fluctuations in output voltage. During charging and discharging of a battery pack, deviations in voltage curves with irregular fluctuations can be spotted due to inconsistencies among cells, which could be enlarged through daily usage, and eventually causes liquid leakage, seal failure, or even thermal run away.

\section{(3) Voltage drop consistency based model}

By comparing the voltage responses among cells before and after excited by electrochemical or mechanical stress, models of this type capture shorttime transient characteristics. In this way, the so called "diving" of a battery cell results from sudden increase 
of internal resistance can be detected to avoid internal short circuit by the end of battery life.

\subsection{Factor Extraction}

All three models in 3.1 have fault diagnosis capabilities, but the characteristics and application scenario of each model are different. Entropy based model can effectively identify risks caused by inconsistencies among cells. Voltage fluctuation based model can accurately quantify changes in voltage fluctuation to realize effective early warning in case of changes in inner structure of cells. Voltage drop based model performs well in capturing abnormal short-term transient effect of power battery. In this paper, the proposed fault diagnosis method is established on top of fusion of multiple models, which realizes fault detection on vehicle level with improved capability for a variety of application scenarios.

\subsubsection{Input Feature Acquisition}

In total, 82 parameters are directly available from the national platform, which can be divided into the following three types. The first type is related to extreme operating conditions of NEVs, such as exceedingly high temperature that may directly lead to thermal runaway or accelerated aging. The second type of parameters characterize performance decline of batteries, including state of health $(\mathrm{SOH})$. The third type of parameters deal with faulty cells, such as the number of abnormal cells diagnosed by the three models, which provides an intuitive understanding of the safety status of the battery pack.

\subsubsection{Characteristic Parameter Screening}

In practice, parameters carry different amount of information. Meanwhile, redundancy exists because of

Table 1: The Selected Features and Definitions

\begin{tabular}{|c|c|}
\hline Selected features & Definition \\
\hline \hline $\begin{array}{c}\text { Output from entropy } \\
\text { model }\end{array}$ & $\begin{array}{c}\text { For faulty vehicles detected by Shannon } \\
\text { entropy model, record maximum, mean } \\
\text { and variance of the abnormity coefficient. }\end{array}$ \\
\hline $\begin{array}{c}\text { Processed data } \\
\text { based on outputs } \\
\text { from sub-models }\end{array}$ & $\begin{array}{c}\text { Average error rate of battery cells } \\
\text { indicated by sub-models }\end{array}$ \\
\hline \multirow{2}{*}{$\begin{array}{c}\text { Risky operational } \\
\text { scenarios }\end{array}$} & Frequent high-power discharging and \\
\cline { 2 - 2 } charging.
\end{tabular}

overlap among parameters. In this sub-section, Pearson correlation coefficient is used for screening [5], where parameters strongly correlated with faults are selected. According to information theory, the amount of information can be expressed by covariance [6]. In this work, parameters with covariance value below 0.1 are filtered. Eventually, 11 characteristic parameters are screened out, as given in Table 1.

\subsection{Model Fusion}

In this part, 11 characteristic parameters are fused by linear weighting, where weighting is done to ensure diagnostic accuracy using Random Forest-Analytic Hierarchy Process (RF-AHP) algorithm. In this way, vehicle risk level can be evaluated, where a score can be assigned to each vehicle. Detailed process is as follows.

(1) Score the importance of each parameter

Analytic Hierarchy Process (AHP) is a method that combines qualitative and quantitative methods to calculate weights [7]. The key step of this method is to score the importance of each parameter based on expert experience to construct a judgment matrix with certain subjectivity. To ease establishment of AHP, Random Forest (RF) is adopted to score parameters.

RF takes decision tree as the base learner and uses bagging principle, which is also known as bootstrap aggregation, to process training data sets. There are two ways to measure the importance of features in a random forest algorithm. The first one is to use Gini index as the partition function to calculate Gini importance of features. The second way, which is adopted in this work, is to use out-of-bag observation. The samples that are not selected after self-service sample aggregation are called 'out of bag (OOB)' [8]. OOB also adopts a verification set and can be used to verify the generalization error of the random forest $G$, calculated as

$$
E_{\text {oob }}(G)=\frac{1}{N} \sum_{n=1}^{N} \operatorname{err}\left(y_{n}, G_{n}^{-}\left(x_{n}\right)\right)
$$

where, $G_{n}^{-}\left(x_{n}\right)$ is a decision tree that only includes $x_{n}$ as OOB.

Essentially, to use OOB data to measure the importance of feature vectors is to reorder the features on OOB samples, where feature ranking can be performed by calculating permutation importance. In this work, permutation importance is used to replaces 
$\left\{x_{n, j}\right\}$ in the original characteristic OOB sample with randomly rearranged $\left\{x_{n, j}\right\}_{n=1}^{N}$ to form a new OOB sample. $V I^{(t)}\left(x_{j}\right)$ is recorded as the importance of the characteristic variable $x_{j}$ obtained from the decision tree $g_{t}$, so we have

$V I^{(t)}\left(x_{j}\right)=E_{o o b}(G)-E_{o o b}^{(P)}(G)$

where, $E_{\text {oob }}^{(P)}(G)$ is the error of re-randomly arranged OOB-sample.

Finally, the importance score of each feature is the mathematic expectation of all trees.

$V I\left(x_{j}\right)=\frac{\sum_{t=1}^{n \text { tree }} V I^{(t)}\left(x_{j}\right)}{\text { ntree }}$

(2) Construct AHP discrimination matrix

The relative importance of each parameter can be obtained from step 1 to construct the judgment matrix of the 11 characteristic parameters screened in 3.2.2. The judgment matrix is constructed by Saaty's 1-9 scale method [9], where larger value means greater difference in importance between two parameters.

\section{(3) Consistency test}

Consistency tests are needed for error detection. The sufficient and necessary condition for the consistency of n-order matrix is the maximum eigenvalue $\lambda_{\max }=\mathrm{n}$, so we have

$A w=\lambda w$

where $A$ is the judgment matrix, $\lambda$ is the set of eigenvalues, $w$ denotes eigenvectors.

In practice, slight inconsistency in the judgment matrix is inevitable, but inconsistency above a certain threshold is not acceptable. Specific threshold values are given as in Table 2. And consistency judgment is performed using consistency indicators $(\mathrm{Cl})$ as

$\mathrm{CI}=\frac{\lambda_{\max }-\mathrm{n}}{\mathrm{n}-1}$

where, $\lambda_{\max }$ is the maximum eigenvalue, $\mathrm{n}$ is the dimension of the judgment matrix. Similarly, $\mathrm{RI}$ is the average random consistency index, and its value is shown in Table 2.
Table 2: Value of RI [9]

\begin{tabular}{|c|c|c|c|c|c|c|c|c|}
\hline $\mathrm{n}$ & 4 & 5 & 6 & 7 & 8 & 9 & 10 & 11 \\
\hline $\mathrm{RI}$ & 0.90 & 1.12 & 1.24 & 1.32 & 1.41 & 1.45 & 1.49 & 1.51 \\
\hline
\end{tabular}

In this way, consistency ratio (CR) is calculated as

$\mathrm{CR}=\frac{\mathrm{CI}}{\mathrm{RI}}$

A CR value smaller than 0.1 is considered within the allowable range, where normalized eigenvector can be used as the weight vector. Modifications are needed on judgment matrix for the cases with CR larger than 0.1 .

(4) Weight calculation

Weight vectors are obtained by eigenvalue method, and are normalized to get the weight of each parameter.

(5) Risk decision

a. The risk value of each vehicle is calculated by linear weighting combined with the parameters and corresponding weights;

b. Sort the sample set vehicles based on their risk levels;

c. Top $10 \%$ vehicles are labeled as extremely highrisk vehicles.

\subsection{Model Validation and Result Analysis}

5 faulty vehicles and 100 normal vehicles of the same model are selected to verify the proposed method. Scores of vehicles are shown in Figure 2, where 4 faulty vehicles are identified as vehicles with high-risk level. And the detection rate of faulty vehicles reached $80 \%$, which met the expectation. The proposed method failed to capture one faulty vehicle, which suffered from traffic accident as indicated from police report. As can be seen, misjudge rate of the proposed method is $6 \%$. Whether the misjudged vehicles are in high risk level is part of future work.

Through further analysis, it can be found that among the 11 parameters, the weights of two parameters that characterize over temperature and high SOC accounts for more than $40 \%$ of total weights. This indicates failures of vehicles are strongly related to SOC and thermal conditions. By referring to accident analysis reports, the major failure mode is identified as thermal runaway. Retrospective historical data shows 
that excessive temperature difference occurs frequently in those battery packs, and the maximum temperature difference is almost $10^{\circ} \mathrm{C}$. This is consistent with the model diagnosis results. Through the above analysis, the effectiveness and rationality of the model are verified.

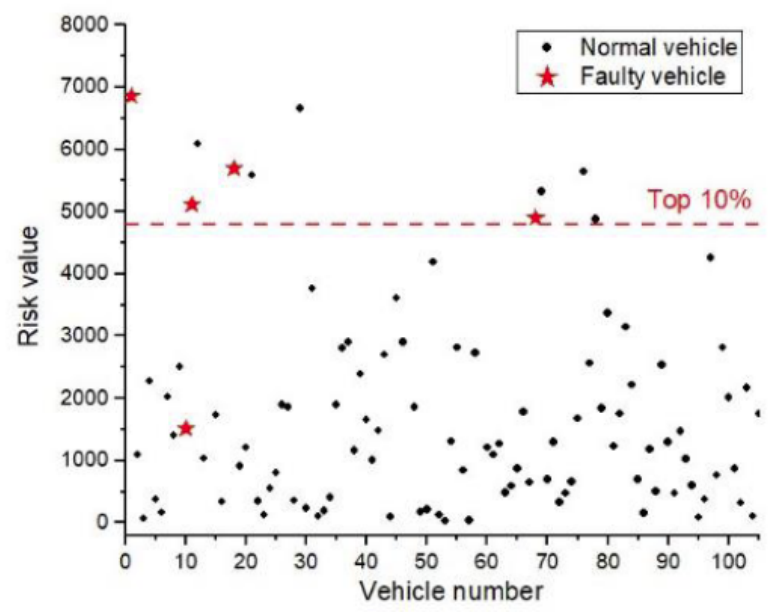

Figure 2: The risk value of each vehicle.

\section{MOTOR}

In this part, permanent magnet synchronous motors are selected as research objectives. Studies are carried out from two aspects, namely motor temperature prediction and over temperature fault diagnosis [10].

\subsection{Motor Temperature Prediction}

Real-time monitoring and accurate prediction of motor temperature are the foundation of fault diagnosis, which is critical to ensure safe operation of NEVs. During vehicle operation, the state parameters of the motor system are non-linear and time-varying, so it is difficult for modeling accurately through experimental and mathematical methods. Fortunately, most of the difficulties can be avoided by combining historical vehicle operational data with environmental information. Temperature prediction is essentially a time series forecasting problem. In this paper, Long Short-Term Memory (LSTM) is adopted as the mathematic tool to solve this problem [11]. Detailed work, including determination of model input parameters, model super parameter optimization, model training and verification, are giving as following.

\subsubsection{Identification of Input Parameters}

Parameters related to motor temperature obtained from the national platform mainly include vehicle speed, motor speed, torque, motor input current, motor input voltage, input power and output power. According to location of the buses in this study, corresponding meteorological information is also included and integrated with vehicle operational data.

In the training process of a LSTM model, more input parameters may lead to worse performance. Basically, parameters with weaker correlation could contribute negative, producing opposite training effect while increasing chances of over fitting. Therefore, the first step is to extract parameters with strong influences, which can be done through calculation of Pearson correlation as shown in Figure 3.

As an illustrative example, the correlation between torque and motor temperature is only 0.143 , whereas much stronger correlation can be observed between vehicle speed and motor speed. In this way, motor speed, input current, input voltage; motor temperature, air temperature and precipitation are selected as inputs to LSTM model.

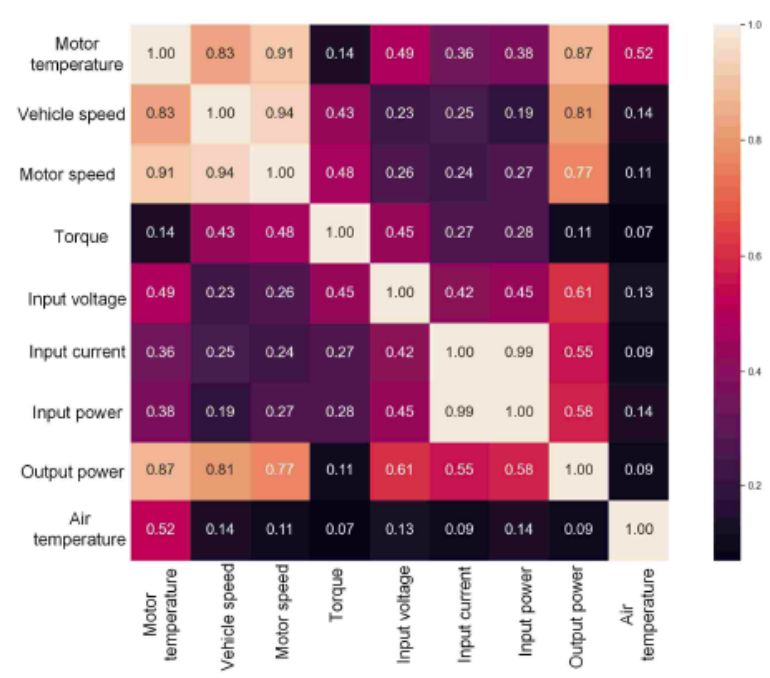

Figure 3: Pearson correlation matrix heatmap.

\subsubsection{Optimization of the Hyper-Parameters}

With selected parameters, the batch size (BS), sliding window length (SWL), number of iterations and other key hyper-parameters of the model need to be optimized before a LSTM model can be trained properly. Correct choice of BS is to find the best balance between memory efficiency and capacity. Adopting a sliding window provides flexibility to reach an optimal tradeoff between learning and historical information remembering. Using appropriate number of iterations helps to obtain better accuracy with reasonable training time while avoiding over fitting. In 
this work, control variable method is used to optimize these hyper-parameters, and results are evaluated based on mean relative error (MRE) expressed as

$$
M R E=\frac{1}{n} \sum_{i=1}^{n} \frac{\left|\hat{P}_{i}-P_{i}\right|}{P_{i}} \times 100 \%
$$

where, $\mathrm{n}$ is the number of training samples or test samples, $\widehat{P}_{l}$ and $P_{i}$ represent the predicted value and the actual value, respectively.

The effect of BS on the predictive performance of the model is shown in Figure 4 (a). It can be seen that when $B S=16$, the MRE is the lowest, which means that the predictive performance is at its optimum.

Figure 4 (b) shows the trend of increasing MRE with the increase of SWL. Ideally, a smaller SWL means more historical information remembered, but smaller SWL also means higher requirement on computational power and longer training time. In this specific case, we found error at $S W L=6$ is very close to that at $S W L=1$. This also means the learning ability at $S W L=6$ is similar to that at $S W L=1$, so the SWS parameter equal to 6 is selected. Figure 4 (c) shows that the loss function value of model training has begun to converge when the number of iterations reaches 15 .

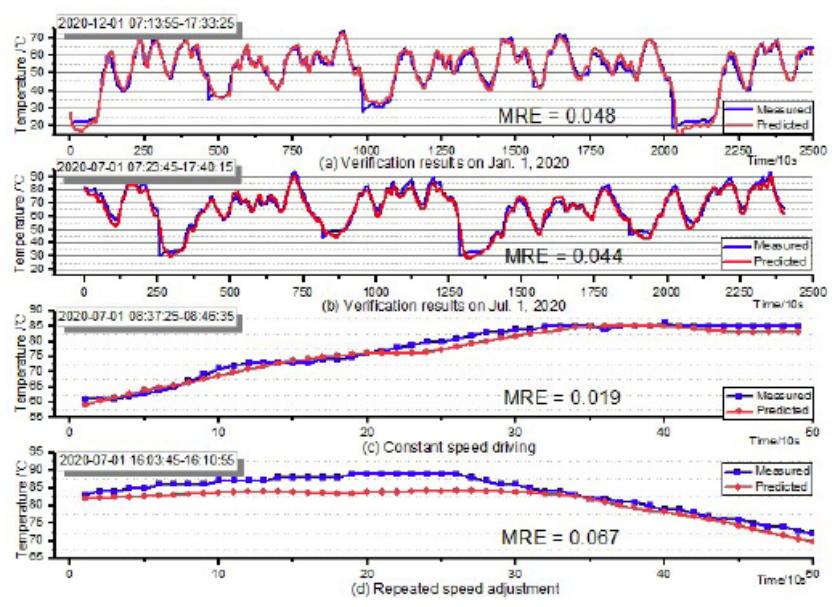

Figure 4: Prediction results of different hyper-parameters.

\subsubsection{Model Training and Verification}

In this sub-section, we take operational data of bus A in 2019 as the training set to establish a LSTM network, which consists of six layers, including one input layer, two hidden layers (80 neurons per layer), one linearly activated fully connected hidden layer and one output layer. Predictive performance of the model is validated with part of the driving data of vehicle $A$ in 2020 .
As can be seen in Figure 5, the predicted values and measured values match well when the driving patterns are relatively mild (e.g. constant speed driving state). The forecast bias increases when driving conditions become wild (e.g. repeated speed up and break). This discrepancy is partially attributes to the long data acquisition interval compared with changes in dynamic real-world driving behaviors, which makes it impossible to fully record the change process in training set. Overall, the maximum deviation does not exceed $10^{\circ} \mathrm{C}$ and is still within acceptable range. In addition, in more than $88.3 \%$ of the scenarios, the error does not exceed $5^{\circ} \mathrm{C}$.
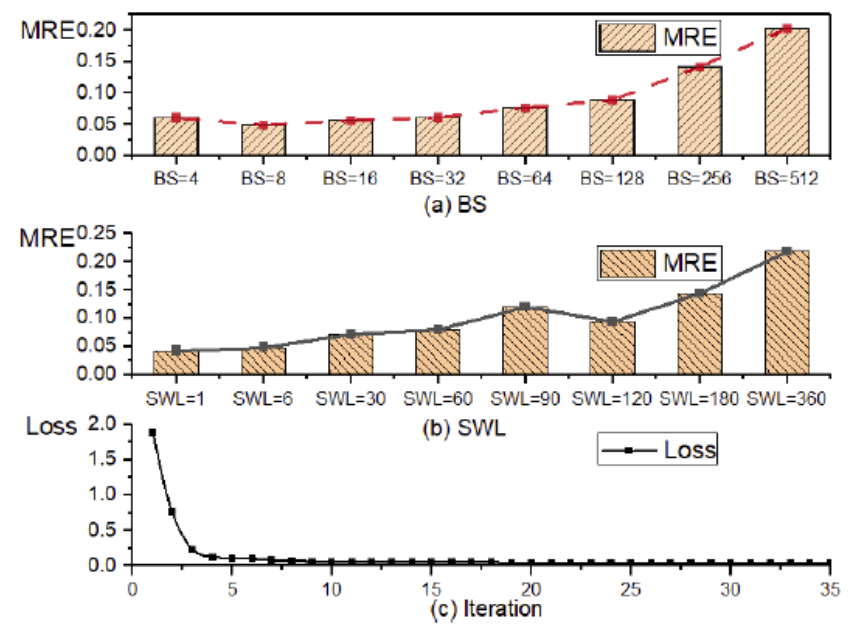

Figure 5: Comparison between measured temperature and model predicted temperature.

\subsection{Motor Over-temperature Diagnosis}

In this part, we take PMSM as the research object to introduce a fault diagnosis model for overtemperature. Model building, model training and verification are covered.

\subsubsection{Parameter Selection and Data Preparation}

Parameters with strong correlation are given in Figure 3, including motor speed, input current, input voltage, motor temperature and air temperature. 1200 cases of motor over-temperature are chosen to establish and verify the proposed model. Samples of both normal and faulty driving cycles are included at 10s sampling interval. The ratio of faulty samples to normal samples is $1: 100$, which constitutes the sample database for model training. For validation purposes, samples are randomly divided into training set and test set based on the ratio of 8:2. 


\subsubsection{Back Propagation Neural Network (BPNN)}

Compared with other artificial intelligence methods, neural network has several favorable features, including high adaptability, strong self-learning ability, fault tolerance, as well as parallel processing capability, thus suitable for NEV applications [12]. In this work, BPNN is used to identify and diagnose motor overtemperature fault as shown in Figure 6.

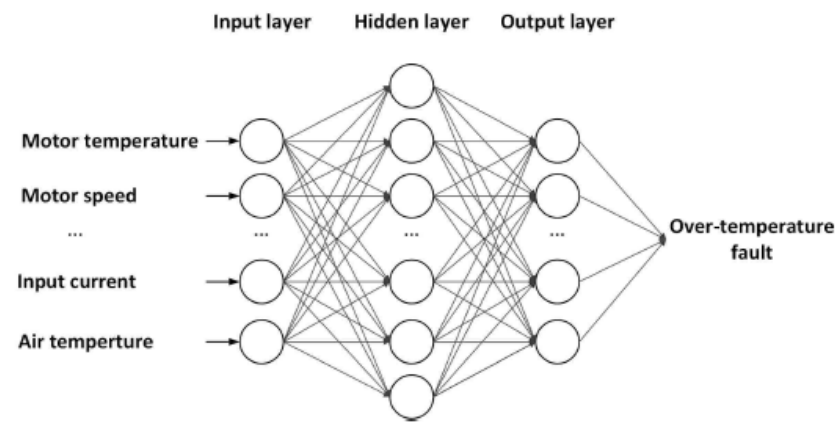

Figure 6: Schematic diagram of motor over-temperature diagnosis neural network model.

\subsubsection{Model Training and Verification}

In this work, the established BP neural network has 2 hidden layers with 100 neurons per layer, learning rate and number of iterations are set as 0.01 and 500 , respectively.

The training process of the model is divided into three steps. Firstly, the input and output values of each layer are calculated in sequence from the input layer, and the output values are passed to a SoftMax classifier. Secondly, the cross error function is used to calculate the loss of the target result and the output from the SoftMax classifier. Finally, gradient descent algorithm is used to adjust the connection weight between neurons in each layer so that the loss function changes in the direction of reduction. Steps given above are repeated until model accuracy meets the requirements. Loss function values and accuracy changes during training are shown in Figure 7.

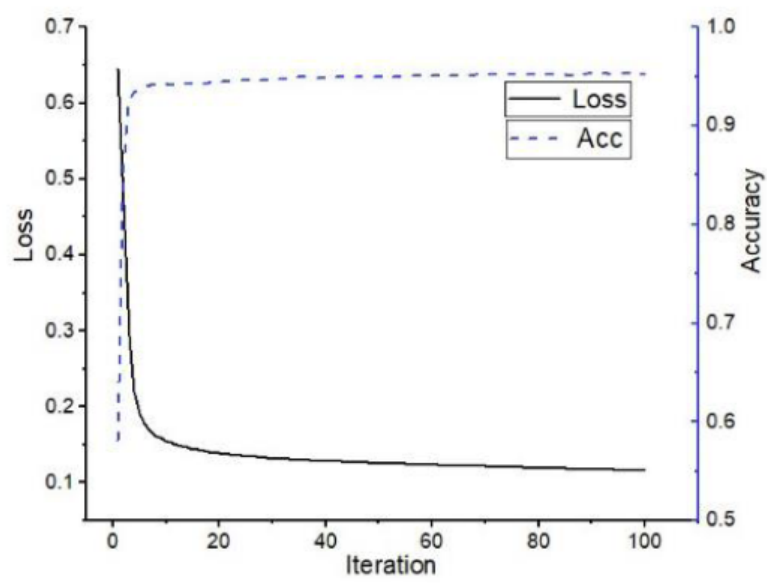

Figure 7: Changes in loss and accuracy during model training.

In validation phase, samples with motor overtemperature fault are defined as positive examples, whereas those without fault are defined as negative examples. For verification, true positives indicate the number of samples that are actually positive and identified as positive. A false positive is the case where a sample is actually negative but misjudged as positive. Similar rule also applies for true negative and false negatives. In this way, accuracy, precision and recall rate can be calculated as

$$
\begin{aligned}
& \text { Accuracy }=\frac{\mathrm{TP}+\mathrm{TN}}{\mathrm{TP}+\mathrm{FP}+\mathrm{FN}+\mathrm{TN}}=99.67 \% \\
& \text { Precision }=\frac{\mathrm{TP}}{\mathrm{TP}+\mathrm{FP}}=85.77 \% \\
& \text { Recall }=\frac{\mathrm{TP}}{\mathrm{TP}+\mathrm{FN}}=80.41 \%
\end{aligned}
$$

As can be seen, accuracy, precision, and recall rate are $99.67 \%, 85.77 \%$ and $80.41 \%$, respectively, which indicate that the model has good over-temperature fault diagnosis ability.

\section{ELECTRONIC CONTROL SYSTEM}

Electric control system is the brain of NEVs. Fortunately, failure rate of a control system is much

Table 3: Test Set and Prediction Results

\begin{tabular}{|c|c|c|c|}
\hline & Pred1 & Pred0 & $\Sigma$ \\
\hline \hline Real1 & $\mathrm{TP}=193$ & $\mathrm{FN}=47$ & $N_{+}=240$ \\
\hline Real0 & $\mathrm{FP}=32$ & $\mathrm{TN}=23968$ & $N_{-}=24000$ \\
\hline$\Sigma$ & $\widehat{N_{+}}=225$ & $\widehat{N_{-}}=24015$ & $\mathrm{~N}=24240$ \\
\hline
\end{tabular}


lower than that of a battery system. Furthermore, comprehensive study of electric control systems requires detailed data directly from controllers such as CAN bus signals, which is not available from the national platform. For these two reasons, in this part, unlike deterministic methods for batteries and motors, we establish a supplementary model for fault diagnosis that provides suggestion on whether electronic control systems are in proper condition.

\subsection{Model Input Characteristic Parameters and Data Preprocessing}

Through data screening on national platform, four parameters are identified to be related safety of the electronic control systems, namely insulation resistance alarm, DC/DC temperature alarm, DC/DC status alarm and hazardous voltage interlock loop alarm. In practice, false alarm can be generated during information collection and transmission, which has serious impact for model accuracy. In this work, false alarms are identified and filtered based on frequency and duration as shown in Figure 8.

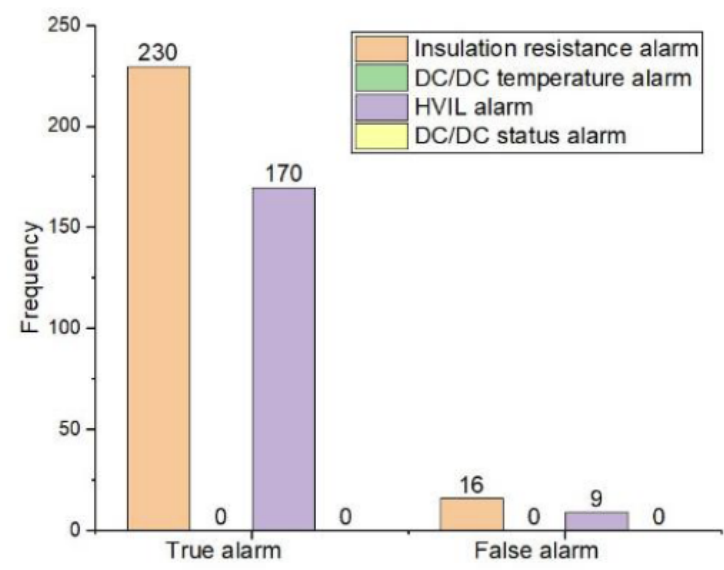

Figure 8: True and false alarm data statistics.

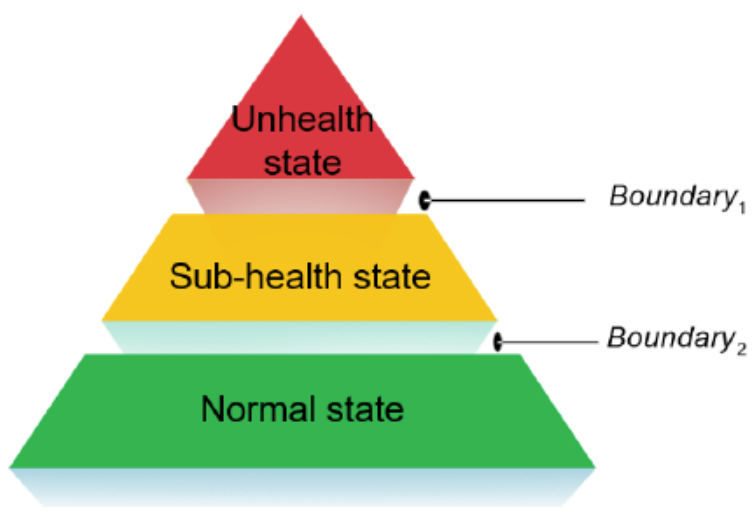

Figure 9: Diagram of health status classification of electrical control system.

\subsection{Health Eigenvalue Calculation}

Frequency and duration of alarms are further processed through Z-score standardization, where dimensions of the two characteristic parameters are unified and integrated into health state eigenvalues, expressed as

$T_{i}=\frac{t_{i}-\mu_{t}}{\sigma_{t}}$

$C_{i}=\frac{c_{i}-\mu_{c}}{\sigma_{c}}$

$E_{i}=T_{i}+C_{i}$

where $t_{i}$ and $C_{i}$ stand for alarm duration and frequency from the $i_{t h}$ vehicle, $\mu_{t}$ and $\mu_{c}$ are mean values whereas $\sigma_{c}$ and $\sigma_{t}$ denote standard deviations. In the end, $E_{i}$ is the calculated health state eigenvalue.

\subsection{Quantification of Electronic Control System Health}

Health status of electrical control systems are classified in a statistical method, where three states are defined, namely normal state, sub-health state and unhealth state. Two boundaries shall be defined among the three states. The value of the boundary between sub-health and unhealth is determined by the Laida criterion, given as

Boundary $_{1}=\mu_{E}+3 * \sigma_{E}$

where $\mu_{E}$ is the mean value of samples $E_{i}(i=1,2,3, \ldots, 80)$, and $\sigma_{E}$ is the standard deviation of sample $E_{i}(i=1,2,3, \ldots, 80)$. In this case, Boundary equals to $3 \sqrt{2}$.

The second boundary is between normal state and sub-healthy state, and is calculated by means of Boxplot as [13]

$I Q R=Q_{3}-Q_{1}$

Boundary $_{2}=Q_{3}+1.5 * I Q R$

where $Q_{3}$ and $Q_{1}$ is the upper and lower quartile of sample $E_{i}(i=1,2,3, \ldots, 80)$, and IQR is the interquartile range. In this way, Boundary $y_{2}$ is equal to 2.0415 .

\subsection{Results and Analysis}

Evaluations are performed over 80 vehicles, where the probability density distribution of electrical control 
health is given in Figure 10. As can be seen in Figure 11 , the health status statistics of the electronic control system show that $86.25 \%$ of the total samples are in normal state, $6.25 \%$ in sub-healthy state and $7.5 \%$ in unhealthy state.

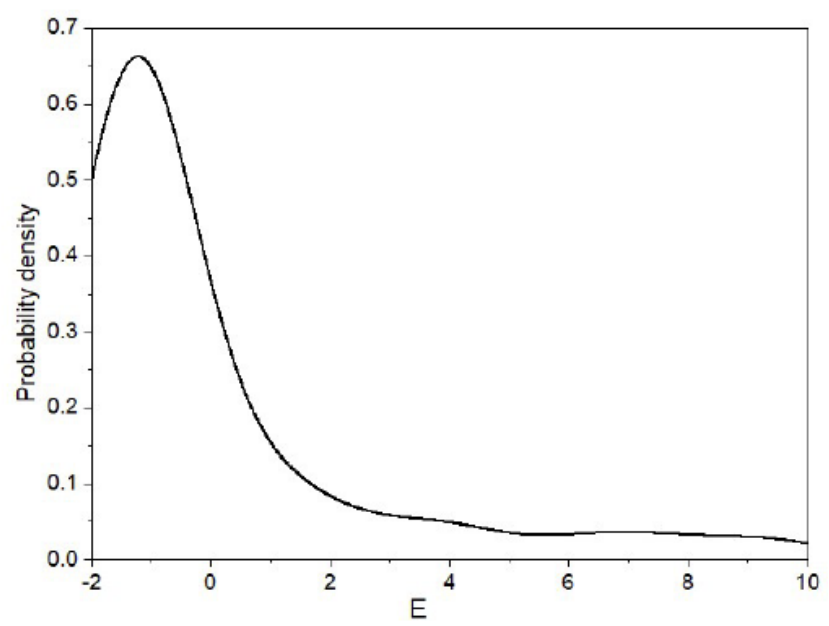

Figure 10: Probability density distribution curve of $E_{i}$.

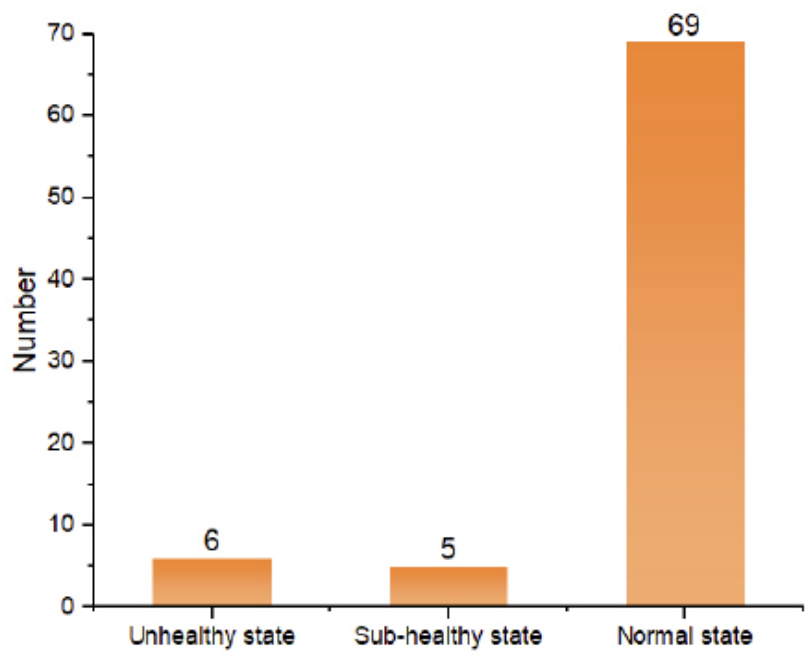

Figure 11: Health status statistics of the electronic control system.

\section{CONCLUSION}

In this work, we proposed a system of methods that can diagnose faults in key components of NEVs. Validity and practicability of models are verified by realworld operational data. Results show the proposed framework contributes in improving overall safety level. In current stage of study, due to limitations in measurable variables and relatively poor quality of data, there are still some flaws or compromises in model establishment. In the follow-up study, experiments shall be done as the compensation to further improve accuracy of each subsystem model.

\section{REFERENCES}

[1] Yang Zhao, Zhenpo Wang, Lei Zhang, Fault and defect diagnosis of battery for electric vehicles based on big data analysis methods. Applied Energy. https://doi.org/10.1016/j.apenergy.2017.05.139

[2] Feng Xuning, Zheng Siqi, Ren Dongsheng, et al. Investigating the Thermal Runaway Mechanisms of Lithiumion Batteries Based on Thermal Analysis Database [J]. Appled Energy, 2019; 246: 53-64. https://doi.org/10.1016/j.apenergy.2019.04.009.

[3] Zhenyu Sun, Peng Liu, Zhenpo Wang, Real-time Fault Diagnosis Method of Battery System Based on Shannon Entropy, Energy Procedia, 2017; 105: 2354-2359, ISSN 1876-6102. https://doi.org/10.1016/j.egypro.2017.03.676.

[4] Zhenpo Wang, Jichao Hong, Peng Liu and Lei Zhang. Voltage Fault Diagnosis and Prognosis of Battery Systems Based on Entropy and Z-score for Electric Vehicles. Applied Energy. 2017; 196: 89-302. https://doi.org/10.1016/j.apenergy.2016.12.143.

[5] Jingwen Song, Pengfei Wei, Marcos A. Valdebenito, Matthias Faes, Michael Beer, Data-driven and active learning of variance-based sensitivity indices with Bayesian probabilistic integration, Mechanical Systems and Signal Processing, Volume 163, 2022, 108106, ISSN 08883270.

[6] Imane Jebli, Fatima-Zahra Belouadha, Mohammed Issam Kabbaj, Amine Tilioua, Prediction of solar energy guided by pearson correlation using machine learning, Energy, Volume 224, 2021, 120109, ISSN0360-5442. https://doi.org/10.1016/j.energy.2021.120109

[7] Lee, Sangwook (2015). Determination of Priority Weights under Multiattribute Decision-Making Situations: AHP versus Fuzzy AHP. Journal of Construction Engineering and Management, 141(2): 05014015 https://doi.org/10.1061/(asce)co.1943-7862.0000897

[8] BYLANDER T. Estimating generalization error on two-class datasets using out-of-bag estimates [J]. Machine Learning 2002; 48(1-3): 287-297 RF

[9] Saaty T L. A scaling method for priorities in hierarchical structures [J]. Journal of Mathematical Psychology, 1977(15): 234-281.

[10] Meinguet F, Kestelyn X, Semail E, et al. Fault Detection, Isolation and Control Reconfiguration of Three-Phase PMSM Drives [J]. 2011 IEEE International Symposium on Industrial Electronics (ISIE) https://doi.org/10.1109/ISIE.2011.5984483

[11] Jichao Hong, Zhenpo Wang, Wen Chen and Yongtao Yao. Synchronous multi-parameter prediction of battery systems on electric vehicles using long short-term memory networks. Applied Energy, 2019; 254: 113648. https://doi.org/10.1016/j.apenergy.2019.113648.

[12] Wang Lin, Zeng Yi, Chen Tao. (2015). Back propagation neural network with adaptive differential evolution algorithm for time series forecasting. Expert Systems with Applications, 42(2): 855-863.

https://doi.org/10.1016/j.eswa.2014.08.018

[13] Yin Hao, Wang Zhenpo, Liu Peng, Zhang Zhaosheng, Li Yang. Voltage Fault Diagnosis of Power Batteries based on Boxplots and Gini Impurity for Electric Vehicles [C] 2019 
Electric Vehicles International Conference (EV), Bucharest,

https://doi.org/10.1109/EV.2019.8892849

Romania 2019: 1-5.

Received on 02-12-2021

Accepted on 19-12-2021

Published on 30-12-2021

DOI: https://doi.org/10.31875/2409-9848.2021.08.8

(c) 2021 Wang et al.; Zeal Press.

This is an open access article licensed under the terms of the Creative Commons Attribution Non-Commercial License

(http://creativecommons.org/licenses/by-nc/3.0/), which permits unrestricted, non-commercial use, distribution and reproduction in any medium, provided the work is properly cited. 